\title{
Validação de metodologia espectrofotométrica para quantificação dos flavonóides de Bauhinia cheilantha (Bongard) Steudel
}

\author{
Tadeu José da Silva Peixoto Sobrinho ${ }^{1}$, Carlos Henrique Tabosa Pereira da Silva', \\ João Eudes do Nascimento', Júlio Marcelino Monteiro², Ulysses Paulino de Albuquerque ${ }^{2}$, \\ Elba Lúcia Cavalcanti de Amorim ${ }^{*}$ \\ 'Laboratório de Produtos Naturais, Departamento de Ciências Farmacêuticas, Centro de Ciências da Saúde, \\ Universidade Federal de Pernambuco \\ 2Laboratório de Etnobotânica Aplicada, Departamento de Biologia, Área de Botânica, \\ Universidade Federal Rural de Pernambuco
}

*Correspondência:

E.L.C. de Amorim

Departamento de Ciências

Farmacêuticas - Centro de Ciências da

Saúde - Universidade Federal de

Pernambuco - Av. Prof Arthur de Sá,

$\mathrm{s} / \mathrm{n}$, Cidade Universitária

54740-521 - Recife - PE, Brasil

E-mail: elba@ufpe.br
Os laboratórios de fitoterápicos necessitam de metodologias que assegurem o controle de qualidade de seus produtos quando os mesmos não constam em farmacopéias ou monografias oficiais. Baseando-se neste fato, o presente estudo visou validar uma metodologia analítica para quantificação de flavonóides contidos nos extratos da pata-de-vaca (Bauhinia cheilantha [Bongard] Steudel), através de espectrofotometria no visivel, como equivalentes de Rutina $(\mu \mathrm{g} / \mathrm{mL})$. Todos os parâmetros exigidos pela ANVISA foram avaliados. No teste de especificidade, observou-se a existência de pico máximo a $420 \mathrm{~nm}$. O método foi considerado linear e com alta sensibilidade de quantificação $(2,51 \mu \mathrm{g} / \mathrm{mL})$. O método também mostrou-se robusto e com elevada recuperação (98,36\%). Os resultados obtidos para repetibilidade (intra-corrida), precisão intermediária (inter-corridas) e reprodutibilidade certificaram a precisão do método com valores entre 0,31 e 3,58\%, sendo também considerado exato (95,71-105,50\%). Com este estudo, o método proposto foi considerado especifico, preciso, reprodutivel, exato, de baixo custo e fácil execução.

\section{INTRODUÇÃO}

O gênero Bauhinia (Caesalpiniaceae) possui aproximadamente 300 espécies e, no Brasil, podemos encontrar cerca de 20\% destas em seu território (Haver, 2002; Silva, Cechinel Filho, 2002), muitas presentes na composição de diversos fitoterápicos ou comercializadas em feiras livres (Melo et al., 2004). Apesar de ser bastante empregada na medicina popular (Albuquerque, Andrade, 2002a,b;
Albuquerque et al., 2005), o gênero Bauhinia até o momento não faz parte da Farmacopéia Brasileira (Melo et al., 2004).

Bauhinia cheilantha (Bongard) Steudel é uma espécie amplamente usada na medicina tradicional em diversas comunidades rurais, podendo ser encontrada em quintais agroflorestais ou em áreas de mata de caatinga (Albuquerque, Andrade, 2002a). É uma espécie de expressiva importância local sendo usada na produção de remé- 
dios tradicionais com ação antiinflamatória, antidiabética, para distúrbios digestivos, reumatismo e sedativa (Albuquerque et al., 2005; Almeida et al., 2005). Todavia, ainda são escassos os estudos que investiguem sua atividade biológica. Por exemplo, o efeito hipoglicemiante da fração butanólica do extrato foliar foi testado em ratos diabéticos induzidos por aloxano (Silva, Cechinel Filho, 2002).

Outras espécies do gênero Bauhinia, por sua vez, já foram os objetivos de diferentes investigações. Argolo et al. (2004) estudando a ação antioxidante das folhas de Bauhinia monandra Kurz., mostrou que os extratos clorofórmico e em acetato de etila, apresentaram excelente atividade, com concentração de inibição $\mathrm{IC}_{50}$ de $2 \mathrm{mg} / \mathrm{g}$ de 1,1-difenil-2-picril-hidrazil (DPPH) e porcentagem de inibição IP de $60-65 \%$. Esta atividade foi atribuída aos esteróides e flavonóides presentes nas folhas, indicando potente atividade antioxidante quando comparada ao grupo tratado com catequinas e com outros extratos vegetais.

A ação antidiabética de diferentes frações do extrato foliar de Bauhinia candicans Benth. foi avaliada por Fuentes et al. (2004). O tratamento utilizando doses de $8 \mathrm{mg} / \mathrm{kg}$ da fração III (Canferol-3-O-rutinosídeo) reduziu, significativamente, os níveis de glicemia, evidenciando uma atividade mais pronunciada. Estes resultados sugerem que os extratos de $B$. candicans aumentam o metabolismo da glicose e que podem conter princípios ativos com propriedades hipoglicemiantes.

Durante os últimos anos, diversos fitoterápicos que possuem em sua constituição plantas do gênero Bauhinia, tiveram seu registro indeferido por estar em desacordo com a legislação vigente (Brasil, 2007). Para a regularização de fitoterápicos, a Agência Nacional de Vigilância Sanitária (ANVISA) exige através de sua Resolução da Diretoria Colegiada (R.D.C) n 48, de 16 de março de 2004 (Brasil, 2004), entre outras recomendações, a validação da metodologia analítica para registro do produto acabado.

A Resolução Específica (R.E.) nº 899, de 29 de maio de 2003, "Guia para validação de métodos analíticos e bioanalíticos" (Brasil, 2003) da ANVISA e a norma Q2(R1), "Validation of analytical procedures: text and methodology" do International Conference on Harmonisation - ICH (ICH, 1996), determinam que para validar uma metodologia, faz-se necessário avaliar os seguintes parâmetros: especificidade, linearidade, robustez, limite de detecção (LD), limite de quantificação (LQ), precisão e exatidão. Por tanto, a validação de metodologias analíticas necessita ser específica, robusta, sensível, precisa e exata, constituindo fundamental importância para o controle de qualidade dos produtos e sendo parte das normas de Boas Práticas de Fabricação e Controle (Pimentel, Barros Neto, 1996; Barros Neto et al., 2002).
Em virtude das necessidades normativas no que se refere à regularização de fitoterápicos, o enfoque deste trabalho foi validar uma metodologia por espectrofotometria capaz de quantificar os constituintes flavônicos presentes nas folhas de Bauhinia cheilantha (Bongard) Steudel, para que possa ser usada rotineiramente nos laboratórios de controle de qualidade.

\section{MATERIAL E MÉTODOS}

\section{Equipamentos}

Empregaram-se espectrofotômetro GBC UV/Visível 911A (Melbourne), cubeta de quartzo de $10 \mathrm{~mm}$ de caminho óptico (Equilab), placa de aquecimento (Tecnal TE018) e triturador industrial (Bermar BM30) para validar o método proposto. Em todos os parâmetros foram utilizadas balanças e vidrarias analíticas.

\section{Reagentes e padrão de referência}

Os solventes usados para quantificar os flavonóides de $B$. cheilantha (Bongard) Steudel foram todos de grau analítico: metanol p.a. Cinética Reagentes \& Soluções e Dinâmica Reagentes Analíticos, ácido acético glacial p.a. Merck, cloreto de alumínio 99,5\% Vetec Química Fina e piridina Vetec Química Fina 99,0\%. Como padrão para flavonóides, foi utilizado Rutina (Merck), com grau de pureza de $99,5 \%$.

\section{Amostra vegetal}

As folhas de $B$. cheilantha foram obtidas na Estação Experimental do Instituto Agronômico de Pernambuco IPA ( $08^{\circ} 14^{\prime} 18,2^{\prime \prime}$ S e $\left.35^{\circ} 54^{\prime} 57,1^{\prime \prime}\right)$, situado no agreste pernambucano, sendo a identificação confirmada pelo Prof. Dr. Ulysses P. de Albuquerque, do Departamento de Biologia da Universidade Federal Rural de Pernambuco e a exsicata foi depositada no Herbário Prof. Vasconcelos Sobrinho do Departamento de Botânica, na Universidade Federal Rural de Pernambuco, sob o n ${ }^{\circ} 49684$. Após secagem à temperatura ambiente, as folhas foram pulverizadas em triturador industrial para subseqüente análise dos parâmetros de validação.

\section{Procedimentos Experimentais}

O padrão (Rutina) foi preparado em balão volumétrico de 100,0 mL com metanol, obtendo-se concentração final de $0,5 \mathrm{mg} / \mathrm{mL}$. A cada balão foram acrescentados $0,6 \mathrm{~mL}$ de ácido acético glacial, $10,0 \mathrm{~mL}$ da solução metanólica de 
piridina $20 \%$ e $2,5 \mathrm{~mL}$ do reagente cloreto de alumínio em metanol 50,0 mg/L, completando-se o volume com água destilada. Após 30 minutos em temperatura ambiente, foram realizadas as leituras em espectrofotômetro a $420 \mathrm{~nm}$, utilizando-se todos os reagentes anteriores (exceto extrato ou padrão) como solução-branco para zerar o aparelho.

Da amostra vegetal seca e pulverizada, $500,0 \mathrm{mg}$ foram pesados e transferidos para Erlenmeyers de 50,0 mL, sendo adicionados $25,0 \mathrm{~mL}$ de metanol e aquecidos sob ebulição branda em placa de aquecimento por 30 minutos. Os extratos foram filtrados em papel de filtro e transferidos quantitativamente para balões volumétricos de $50,0 \mathrm{~mL}$. O resíduo do material foi lavado com $25,0 \mathrm{~mL}$ de metanol e novamente filtrado para o balão, tendo o volume completado com metanol. Do extrato, $1,0 \mathrm{~mL}$ foi transferido para balão volumétrico de $25,0 \mathrm{~mL}$ e, de forma semelhante ao procedimento empregado para a construção da curva com o padrão, foram acrescentados os mesmos reagentes. Após 30 minutos em temperatura ambiente foram realizadas as leituras em espectrofotômetro a $420 \mathrm{~nm}$ em cubetas de quartzo.

\section{Parâmetros de validação}

O ensaio de especificidade foi conduzido com os extratos metanólicos das amostras foliares de $B$. cheilantha pulverizadas e Rutina como padrão, ambos à concentração de $18,0 \mu \mathrm{g} / \mathrm{mL}$, para verificação de possíveis interferentes. Os espectros de absorbância foram realizados na faixa compreendida entre $300-500 \mathrm{~nm}$ e forneceram os dados para construção do gráfico de especificidade.

Para a linearidade foi usada a média de três intervalos com repetições autênticas, que contemplavam seis concentrações da solução de Rutina $0,5 \mathrm{mg} / \mathrm{mL}(6,0,8,0,10,0$, $12,0,16,0$ e $20,0 \mu \mathrm{g} / \mathrm{mL})$. Após relação linear visual, os resultados foram analisados estatisticamente para definir o coeficiente de determinação (mínimo aceitável $\mathrm{R}^{2}=0.99$ ), a equação de regressão, o ajuste linear e o desvio padrão relativo (Barros Neto et al., 2002; Brasil, 2003).

Os limites de detecção e quantificação foram estimados (em $\mu \mathrm{g} / \mathrm{mL})$ considerando o desvio padrão em razão ao coeficiente angular (inclinação da reta) obtidos pela linearidade, nos quais foram utilizadas as equações 1 e 2 para determinar o limite de detecção e quantificação, respectivamente (Brasil, 2003).

$$
\begin{aligned}
& \mathrm{LD}=\mathrm{DPa} \times 3 / \mathrm{IC} \\
& \mathrm{LQ}=\mathrm{DPa} \times 10 / \mathrm{IC}
\end{aligned}
$$

Em que o LD é o limite de detecção; LQ é o limite de quantificação; DPa é o desvio-padrão relativo; IC é a inclinação de curva de calibração.
O parâmetro recuperação foi realizado com três amostras em triplicata e o resultado foi obtido através da equação 3 .

$\mathrm{R}(\%)=\frac{\mathrm{CTF}-\mathrm{CFE}}{\mathrm{CFP}} \times 100$

CTF compreende a concentração total de flavonóides (padrão Rutina adicionado ao extrato foliar de $B$. cheilantha), CFE compreende a concentração de flavonóides no extrato foliar e CFP a concentração de flavonóides do padrão Rutina. R (\%) é a recuperação obtida (Brasil, 2003).

Os fatores a serem considerados para analisar a robustez foram: tempo de extração (20 e 40 minutos), estabilidade de leitura (20 e 40 minutos) e diferentes fabricantes de solventes (Marca 1 e Marca 2), o que está de acordo com o preconizado pela ANVISA (Brasil, 2003). Os resultados foram avaliados mediante comparação feita através da análise de variância.

Os ensaios de repetibilidade e precisão intermediária foram determinados por seis amostras de mesma concentração $(18,0 \mu \mathrm{g} / \mathrm{mL})$ executados no mesmo dia (intra-corrida) e dois dias consecutivos por analistas diferentes (inter-corrida) (Brasil, 2003). Os resultados foram expressos como desviopadrão relativo (Equação 4). A reprodutibilidade foi realizada no Laboratório de Tecnologia dos Medicamentos e compreendeu seis amostras de mesma concentração $(18,0 \mu \mathrm{g} / \mathrm{mL})$. A exatidão foi avaliada por três controles (em triplicata) de concentração baixa $(9,0 \mu \mathrm{g} / \mathrm{mL})$, média $(18,0 \mu \mathrm{g} / \mathrm{mL})$ e alta $(27,0 \mu \mathrm{g} / \mathrm{mL})$. A exatidão (Equação 5) foi calculada, individualmente para cada controle.

$$
\mathrm{DPR}(\%)=\mathrm{DP} / \mathrm{CMD} \times 100
$$$$
\mathrm{E}(\%)=\mathrm{CME} / \mathrm{CT} \times 100
$$

Em que o DPR (\%) é a precisão; DP é o desvio-padrão; CMD é a concentração média determinada; $\mathrm{E}(\%)$ é a exatidão; CME é a concentração média experimental; e CT é a concentração teórica.

\section{RESULTADOS E DISCUSSÃO}

\section{Especificidade}

Na Figura 1, podemos observar o espectro obtido para o extrato de $B$. cheilantha $(18,0 \mu \mathrm{g} / \mathrm{mL})$ e para a Rutina $(18,0 \mu \mathrm{g} / \mathrm{mL})$, na faixa compreendida entre 300-500 nm, sendo evidenciado apenas um pico de absorção máxima a $420 \mathrm{~nm}$. Com isso, confirmamos que neste comprimento de onda é possível quantificar especificamente o padrão para flavonóide e os contidos no extrato, mesmo na presença de impurezas. 


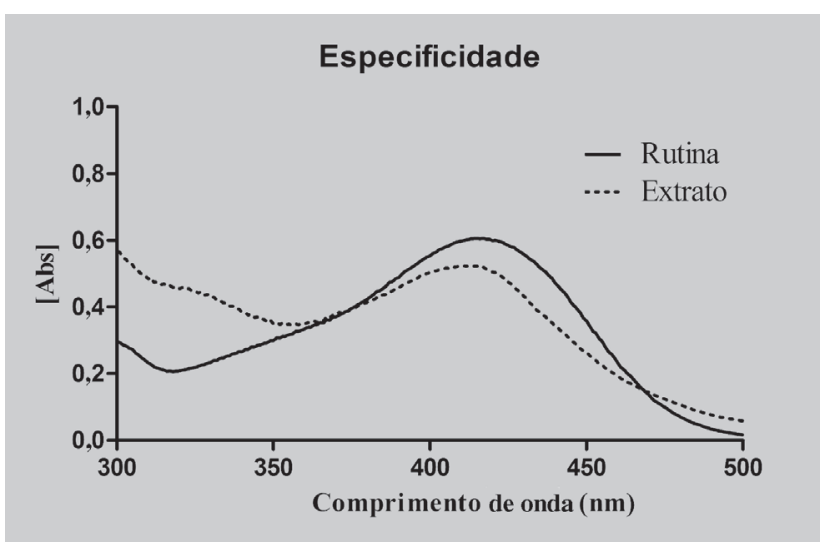

FIGURA 1 - Especificidade do método construído com padrão Rutina $(18,0 \mu \mathrm{g} / \mathrm{mL})$ e com o extrato foliar de Bauhinia cheilantha (Bongard) Steudel $(18,0 \mu \mathrm{g} / \mathrm{mL})$. Amplitude compreendida entre 300-500 nm.

[Abs] = Absorbância.

\section{Linearidade e Limites de Detecção (LD) e Quantificação (LQ)}

O método espectrofotométrico apresentou linearidade a $420 \mathrm{~nm}$ para as concentrações estudadas $(6,0-20,0 \mu \mathrm{g} / \mathrm{mL})$. A equação da regressão linear média obtida a partir de três curvas de calibração, foi $y=0,0256 x$ $+0,0141$, em que $y$ é a absorbância (nm) e $x$ a concentração $(\mu \mathrm{g} / \mathrm{mL})$ em equivalentes de Rutina (Figura 2). O coeficiente de determinação obtido foi $\mathrm{R}^{2}=0,9994$, comprovando a adequação do método ao intervalo avaliado (Brasil, 2003). Os dados de precisão (DPR) e exatidão (E) do intervalo do método são apresentados na Tabela I.

Os valores dos limites de detecção (LD) e quantificação (LQ) estimados pelas equações 1 e 2, foram

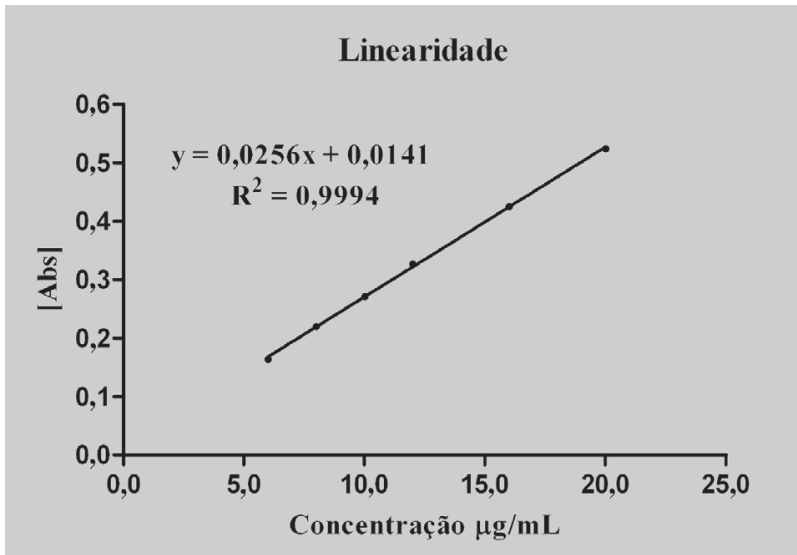

FIGURA 2 - Curva de calibração construída com padrão Rutina $(6,0-20,0 \mu \mathrm{g} / \mathrm{mL})$ a 420 , em que a equação linear média obtida foi $y=0,0256 x+0,0141$.

[Abs] = Absorbância.
TABELA I - Resultados do teste de linearidade por espectrofotometria para flavonóides foliares de Bauhinia cheilantha (Bongard) Steudel, utilizando-se Rutina como padrão

\begin{tabular}{lcccc}
\hline $\begin{array}{l}\text { Concentração } \\
\text { teórica }(\mu \mathrm{g} / \mathrm{mL})\end{array}$ & $\begin{array}{c}\mathrm{C} \\
(\mathrm{n}=3)\end{array}$ & DP & $\begin{array}{c}\text { DPR } \\
(\%)\end{array}$ & $\begin{array}{c}\mathrm{E} \\
(\%)\end{array}$ \\
\hline 6,0 & 6,051 & 0,1033 & 1,71 & 100,85 \\
8,0 & 8,160 & 0,1790 & 2,19 & 102,00 \\
10,0 & 9,957 & 0,2561 & 2,57 & 99,57 \\
12,0 & 12,092 & 0,2151 & 1,78 & 100,77 \\
16,0 & 15,842 & 0,3854 & 2,43 & 99,02 \\
20,0 & 19,658 & 0,5023 & 2,56 & 98,29 \\
\hline
\end{tabular}

$\mathrm{C}=$ Concentração média $(\mu \mathrm{g} / \mathrm{mL})$ de três determinações; $\mathrm{DP}=$ Desvio Padrão; DPR $(\%)=$ Desvio Padrão Relativo (Precisão); E (\%) = Exatidão.

0,75 e 2,51 $\mu \mathrm{g} / \mathrm{mL}$, respectivamente. Com esses resultados, verificamos que o método possui alta sensibilidade para detectar e quantificar o padrão, sem sofrer alteração de fatores intrínsecos do equipamento.

A análise de variância ANOVA (um critério) confirmou que o método proposto foi considerado linear e indica que não há falta de ajuste $(\mathrm{p}<0,05)$ (Pimentel, Barros Neto, 2002), como pode ser visto na Tabela II.

TABELA II - Resultados da Análise de Variância ANOVA (um critério) e teste de ajuste linear $(\mathrm{p}<0,05)$

\begin{tabular}{lccccc}
\hline ANOVA & & & & & \\
Fonte & SQ & gL & MQ & $\mathrm{F}$ & $\mathrm{F}_{\text {Crítico }}$ \\
\hline Modelo & 0,2684 & 1 & 0,2684 & 1625,90 & 4,4940 \\
Residual & 0,0026 & 16 & 0,0002 & Curva Linear \\
Falta de ajuste & 0,0002 & 4 & 0,0000 & 0,2050 & 3,2592 \\
Erro puro & 0,0025 & 12 & 0,0002 & Não há falta de ajuste \\
Total & 0,2710 & 17 & 0,0159 & & \\
\hline
\end{tabular}

\section{Recuperação e Robustez}

A recuperação que mede a eficiência do procedimento de extração do método proposto foi $98,36 \%$, atestando este valor como aceitável (Brasil, 2003).

Os fatores da robustez (tempo de extração, estabilidade e diferentes fabricantes de solventes) foram avaliados em triplicata (Brasil, 2003): intrafatores (parâmetro individual) e interfatores (todos os parâmetros conjuntamente). Os resultados receberam tratamento estatístico pela Análise de Variância ANOVA (dois critério) fator duplo e esta análise se encontra na Tabela III. 
TABELA III - Análise de variância ANOVA (dois critérios) demonstrando que o método possui robustez $(\mathrm{p}>0,05)$.

\begin{tabular}{|c|c|c|c|c|c|}
\hline $\begin{array}{l}\text { ANOVA } \\
\text { Fonte de } \\
\text { variação }\end{array}$ & SQ & $\mathrm{gL}$ & MQ & $\mathrm{F}$ & $\mathrm{F}_{\text {crítico }}$ \\
\hline Entre grupos & 2,4412 & 5 & 0,4882 & 1,7273 & 3,1058 \\
\hline $\begin{array}{l}\text { Dentro dos } \\
\text { grupos }\end{array}$ & 3,3919 & 12 & 0,2826 & & \\
\hline Total & 5,8331 & 17 & & & \\
\hline
\end{tabular}

O método mostrou que não há diferença estatística significativa $(p<0,05)$, já que encontrou valores de F menores que os valores de $\mathrm{F}_{\text {crítico }}$, demonstrando assim ser um método robusto em todos os fatores analisados.

\section{Precisão e Exatidão}

Os dados da repetibilidade (intra-corrida), precisão intermediária (inter-corridas), reprodutibilidade e exatidão do método encontram-se na Tabela IV, cujos valores encontraram-se compreendidos entre $0,44 \%$ e $3,58 \%$. Para o ensaio de exatidão foram encontrados resultados entre 95,71$105,50 \%$. A ANVISA regulamenta que os resultados da precisão não podem ultrapassar $5 \%$ e, para exatidão, não deve ser inferior a 95\% (Brasil, 2003). Estes dados confirmam que o método de quantificação por espectrofotometria proposto encontra-se em conformidade com a legislação vigente e apresenta confiabilidade dos resultados.

\section{CONCLUSÃO}

O método proposto apresenta alta especificidade a $420 \mathrm{~nm}$ para o extrato foliar de Bauhinia cheilantha (Bongard) Steudel o que confere confiabilidade na quantificação de flavonóides. Alinearidade confirma que o método é linear e possui alta sensibilidade de quantificação. É um método robusto para os fatores exigidos pela legislação vigente e encontra-se dentro do limite para a recuperação. Os resultados de precisão e exatidão obtidos atestam confiabilidade necessária para o uso desta metodologia em laboratórios de controle de qualidade. O método de quantificação por espectrofotometria a $420 \mathrm{~nm}$, mostrou-se preciso, exato e reprodutível, aliado à acessibilidade e facilidade de execução.

\section{ABSTRACT \\ Validation of spectrophotometric methodology for quantify flavonoid content in Bauhinia cheilantha (Bongard) Steudel}

Phytotherapeutic laboratories require access to methodologies that guarantee quality control for their products when these items are not already registered in pharmacopeias or official monographs. As such, the present study sought to validate analytical methodologies for quantifying natural flavonoids contained in extracts of "pata-de-vaca" (Bauhinia cheilantha [Bong.] Steudel)

TABELA IV - Resultados da repetibilidade, precisão intermediária, reprodutibilidade e exatidão do método espectrofotométrico para o doseamento dos flavonóides contidos nos extratos de Bauhinia cheilantha (Bongard) Steudel

\begin{tabular}{|c|c|c|c|c|c|c|}
\hline Ensaio & $\begin{array}{l}\text { Concentração } \\
\text { teórica }(\mu \mathrm{g} / \mathrm{mL})\end{array}$ & $\mathrm{N}$ & $\mathrm{C}$ & $\mathrm{DP}$ & $\operatorname{DPR}(\%)$ & $\mathrm{E}(\%)$ \\
\hline Repetibilidade & 18,0 & 6 & 18,069 & 0,203 & 1,12 & 100,38 \\
\hline \multirow{4}{*}{$\begin{array}{l}\text { Precisão } \\
\text { intermediária }\end{array}$} & 18,0 & 6 & 18,113 & 0,641 & 3,54 & 100,63 \\
\hline & 18,0 & 6 & 18,087 & 0,573 & 3,17 & 100,48 \\
\hline & 18,0 & 6 & 18,133 & 0,484 & 2,67 & 100,74 \\
\hline & 18,0 & 6 & 18,060 & 0,647 & 3,58 & 100,33 \\
\hline Reprodutibilidade & 18,0 & 6 & 18,990 & 0,083 & 0,44 & 105,50 \\
\hline \multirow[t]{3}{*}{ Exatidão } & 9,0 & 3 & 9,303 & 0,096 & 1,03 & 103,37 \\
\hline & 18,0 & 3 & 17,465 & 0,079 & 0,45 & 97,03 \\
\hline & 27,0 & 3 & 25,843 & 0,0792 & 0,31 & 95,71 \\
\hline
\end{tabular}

$\mathrm{C}=$ Concentração média $(\mu \mathrm{g} / \mathrm{mL})$ das n determinações; DP = Desvio Padrão; DPR (\%) = Desvio Padrão Relativo (Precisão); E (\%) = Exatidão. 
using visible light spectrophotometry and a rutin standard $(\mu \mathrm{g} / \mathrm{ml})$. All of the parameters required by ANVISA were evaluated. The specificity test revealed a maximum absorption peak at $420 \mathrm{~nm}$. The methodology was considered linear and of high quantification sensitivity $(2.51 \mu \mathrm{g} / \mathrm{ml})$. The methodology also proved to be robust, and had high recovery levels $(98.36 \%)$. The results obtained for repeatability, intermediate precision (intraand inter-day), and reproducibility all certified the precision of the method, with values between 0.31 and $3.58 \%$; the methodology was also considered exact (95.71-105.50\%). This study demonstrated that the proposed methodology can be considered specific, precise, reproducible, exact, of low cost, and easy to perform.

Uniterms: Spectrophotometry/method's validation. Rutin/ use. Bauhinia cheilautha/pharmacognosia. Flavonoids/ quantification. Phytotherapics/quality control.

\section{AGRADECIMENTOS}

Os autores gradecem à CAPES (Coordenação de Aperfeiçoamento de Pessoal de Nível Superior) pelo apoio financeiro, ao Instituto Agronômico de Pernambuco (IPA), à Profa. Dra. Miracy M. de Albuquerque do Núcleo de Controle de Qualidade de Medicamentos e Correlatos (NQCMCUFPE) pelos resultados da especificidade, ao Prof. Dr. Pedro José Rolim Neto do Laboratório de Tecnologia dos Medicamentos (LTM-UFPE) pelas análises de reprodutibilidade.

\section{REFERÊNCIAS BIBLIOGRÁFICAS}

ALBUQUERQUE, U. P.; ANDRADE, L. H. C. Conhecimento botânico tradicional e conservação em uma área de Caatinga no Estado de Pernambuco, Nordeste do Brasil. Acta Bot Bras, v.16, n.3, p.273-285, 2002a.

ALBUQUERQUE, U. P.; ANDRADE, L. H. C. Uso de recursos vegetais da Caatinga: o caso do agreste do estado de Pernambuco (Nordeste do Brasil). Interciência, v.27, n.7, p.336-346, 2002b.

ALBUQUERQUE, U. P.; ANDRADE, L. H. C.; SILVA, A. C. O. Use of plant resources in a seasonal dry forest (Northeastern Brazil). Acta Bot Bras, v.19, n.1, p.27-38, 2005 .

ALMEIDA, C. F. C. B. R.; SILVA, T. C. L.; AMORIM, E. L. C.; MAIA, M. B. S.; ALBUQUERQUE, U. P.Life strategy and chemical composition as predictors of the selection of medicinal plants from the caatinga (Northeast Brazil). J Arid Environ, v.62, n.1, p.127-142, 2005
ARGOLO, A. C. C.; SANT'ANA, A. E. G.; PLETSCH, M.; COELHO, C. B. B. Antioxidant activity of leaf extracts from Bauhinia monandra. Bior Tech, v.95, n.2, p.229233. 2004.

BARROS NETO, B.; PIMENTEL, M. F.; ARAÚJO, M. C. U. Recomendações para calibração em química analítica - Parte I. Fundamentos e calibração com um componente (calibração univariada). Quim. Nova, v.25, n.5, p.856$865,2002$.

BRASIL, Agência Nacional de Vigilância Sanitária (ANVISA). R. E, no 899 de 29 de maio de 2003 - Guia para validação de métodos qualitativos e bioanalíticos. Disponível em: http://e-legis.anvisa.gov.br/leisref/public/ showAct.php?mode=PRINT_VERSION\&id=15132. Acesso em: 05 de janeiro 2007.

BRASIL, Agência Nacional de Vigilância Sanitária (ANVISA). Resolução R.D.C. $\mathrm{n}^{\circ} 48$, de 16 de março de 2004 - Dispõe sobre o registro de medicamentos fitoterápicos. Disponível em: http://e-legis.anvisa.gov.br/ leisref/public/showAct.php?id=10230\#.Acesso em: 05 de janeiro de 2007.

BRASIL, Legislação da Agência Nacional de Vigilância Sanitária. (Visalelgis-ANVISA) Disponível em: http:// www.anvisa.gov.br/legis/index.htm. Acesso em: 14 de janeiro 2007.

FUENTES, O.; ARANCIBIA-AVILA, P.; ALARCÓN, J. Hypoglycemic activity of Bauhinia candicans in diabetic induced rabbits. Fitoterapia, v.75, n.6, p.527-532, 2004.

HAVER, N. J. Desenvolvimento, purificação e caracterização de IgG anti lectina de folha de Bauhinia monandra. 2002. 95p.Tese (Doutorado em Ciências Biológicas) Centro de Ciências Biológicas, Universidade Federal de Pernambuco, Recife.

ICH, International Conference on Harmonization. Validation of Analytical Procedures: Text and Methodology Q2(R1). 1996. Disponível em: http://www.ich.org/LOB/media/ MEDIA417.pdf. Acesso em: 05 de janeiro 2007.

MELO, J. G.; NASCIMENTO, V.T.; AMORIM, E. L. C.; LIMA, C. S. A.; ALBUQUERQUE, U. P.Avaliação da qualidade de amostras comerciais de boldo (Peumus boldus Molina), pata-de-vaca (Bauhinia spp.) e ginco (Ginkgo biloba L.). Rev. Bras. Farmacogn., João Pessoa, v.14, n.2, p.11-120, 2004. 
PIMENTEL, M. F.; NETO, B. B. Calibração: Uma revisão para químicos analíticos. Quim. Nova, v.19, n.3, p.268277, 1996.
SILVA, R. L.; CECHINEL FILHO, V.Plantas do Gênero Bauhinia: Composição química e potencial farmacológico. Quim. Nova, v.25, n.3, p.449-454, 2002.

Recebido para publicação em 23 de outubro de 2007 Aceito para publicação em 30 de julho de 2008 